\title{
SPHERICAL QUADRATURE AND INVERSION OF RADON TRANSFORMS
}

\author{
W. R. MADYCH AND S. A. NELSON ${ }^{1}$
}

\begin{abstract}
An equivalence is established between certain aspects of: (i) mechanical quadrature for integration on a sphere; (ii) a ridge function representation problem connected with inversion of Radon transform data.
\end{abstract}

This paper is concerned with the functional equation

$$
G(x)=\sum_{i=1}^{N} h_{i}\left(\left\langle x, u_{i}\right\rangle\right), \quad|x|<1 .
$$

Here $x$ is a variable point in $d$-dimensional space $R^{d}(d \geqslant 2), u_{1}, \ldots, u_{N}$ are given unit vectors in $R^{d},\left\langle x, u_{i}\right\rangle$ denotes the usual inner (dot) product, $|x|=\langle x, x\rangle^{1 / 2}$, and $h_{1}(t), \ldots, h_{N}(t)$ are real-valued functions on the interval $-1<t<1$.

The reason for our interest in (1) is its connection with the convolution method for inverting Radon transform data, for example see $[2,5,8,9]$. For that application, the $h_{i}$ 's should be chosen so that $G$ is a convolution summability kernel (in other words, an approximate Dirac delta function) in a neighborhood of the origin. Following [3] we call the terms on the right-hand side of (1) ridge functions.

Because of results in [4 and 6], we are particularly interested in cases where $G(x)$ depends only on $|x|$. In such cases, there is a close relation between the ridge function representation (1) and mechanical quadrature on the sphere in $R^{d}$; see Theorem 1. To put off certain technicalities, we have confined Theorem 1 to the case where $G$ and $h_{1}, \ldots, h_{N}$ are assumed to be polynomials. As shown by Theorem 3 , this assumption is redundant. For background on mechanical quadrature for spherical integration see [7 and 10]; here we use the notation $S_{d-1}$ for the unit sphere $|x|=1$ in $R^{d}$ and take $\omega_{d-1}=\int_{S_{d-1}} d \omega(u)$ where $d \omega(u)$ denotes the usual Lebesgue measure on $S_{d-1}$.

THEOREM 1. A necessary and sufficient condition for a radial polynomial $G$ to enjoy a representation (1) with polynomials for $h_{1}, \ldots, h_{N}$ is the existence of constants $w_{1}, \ldots, w_{N}$ (quadrature weights) that make

$$
\frac{1}{\omega_{d-1}} \int_{S_{d-1}} p(u) d \omega(u)=\sum_{i=1}^{N} w_{i} p\left(u_{i}\right)
$$

Received by the editors December 3, 1984.

1980 Mathematics Subject Classification. Primary 65D32, 39B40; Secondary 44A15.

${ }^{1}$ Both authors were partially supported by NSF Grant No. MCS-8202147.

(c) 1985 American Mathematical Society $0002-9939 / 85 \$ 1.00+\$ .25$ per page 
valid for all even polynomials $p$ of degree $\leqslant \operatorname{deg} G$. In that case (1) is satisfied with $h_{i}=w_{i} h_{G}$, where $h_{G}(t)=\sum_{n=0}^{(\operatorname{deg} G) / 2}\left(g_{n} / \mu_{n}\right) t^{2 n}$. Here $g_{n}$ denotes the coefficient of $|x|^{2 n}$ in $G(x)$ and $\mu_{n}$ is the constant defined by

$$
\frac{1}{\omega_{d-1}} \int_{S_{d-1}}\langle x, u\rangle^{2 n} d \omega(u)=\mu_{n}|x|^{2 n} .
$$

REMARKs. In the case $d=2$ the relationship between the directions, $u_{1}, \ldots, u_{N}$ and the maximum degree, call it $\nu=\nu\left(u_{1}, \ldots, u_{N}\right)$, of the radial polynomials $G$ which enjoy representation (1) was studied in [6]. The above theorem shows that this same number $\nu$ gives the maximum degree of even polynomials $p$ for which the exact mechanical quadrature formula (2) holds on the circle.

The fact that (2) is sufficient to imply (1) for radial polynomials is implicit in the calculations of $\$ 5$ in [2].

As mentioned above, it need not be assumed that $G$ and $h_{1}, \ldots, h_{N}$ are polynomials; indeed, Theorem 3 and its corollary show that it suffices for (1) to hold in a distributional sense.

Applying (2) with $p=h_{G}$ gives

$$
\int_{S_{d-1}} h_{G}(\langle x, u\rangle) d \omega(u)=\omega_{d-1} G(x)
$$

which may be written in the equivalent form

$$
\omega_{d-2} \int_{-r}^{r} h_{G}(t)\left(1-\frac{t^{2}}{r^{2}}\right)^{(d-3) / 2} \frac{1}{r} d t=\omega_{d-1} G(r e) .
$$

where $r=|x|$ and $x=r e$. Regarding (5) as an integral equation for $h_{G}$ provides an alternative characterization of $h_{G}$.

Using (5) and standard notation for the Gegenbauer and the Jacobi polynomials, it may be verified (e.g. make the change of variable $t=\gamma \sqrt{x}$ in $[1, \S 13.1$, entry (41)] and take $\nu=1 / 2, \mu=(d-1) / 2, \gamma=\sqrt{r})$ that $h_{G}(t)=C_{2 n}^{\lambda}(t), \lambda=\alpha+d / 2, \alpha>$ -1 , corresponds to $G(x)=c_{n} P_{n}^{(\alpha, \beta)}\left(2|x|^{2}-1\right), \beta=(d-2) / 2$. Here $c_{n}$ is a constant that can be determined by taking $x=0$ in (4). These orthogonal polynomials are very convenient bases for numerical work involving $G$ and $h_{G}$. They are also useful (see [5]) for analyzing effects of noisy data.

Proof. For sufficiency, take $p(u)=\langle x, u\rangle^{2 n}$ in (2) and combine with (3) to obtain

$$
\mu_{n}|x|^{2 n}=\sum_{i=1}^{N} w_{i}\left\langle x, u_{i}\right\rangle^{2 n}
$$

for $n=0, \ldots,(\operatorname{deg} G) / 2$. From this it is evident that $h_{i}=w_{i} h_{G}$ satisfies (1).

To prove necessity, let $2 k$ be the degree of $G$. (If $G=0$ there is nothing to prove.) Equating the terms in (1) that are homogeneous of degree $2 k$ gives

$$
c|x|^{2 k}=\sum_{i=1}^{N} a_{i}\left\langle x, u_{i}\right\rangle^{2 k}
$$


where $c$ is the coefficient of $|x|^{2 k}$ in $G(x)$ and $a_{i}$ is the coefficient of $t^{2 k}$ in $h_{i}(t)$. Applying the Laplacian to both sides of (6) and dividing by $2 k(2 k-1)$ gives an equation of the same form with a new $c$, the same $a_{i}$ 's and $k$ replaced by $k-1$. Repeating this process we see there are constants $c=c_{k}, \ldots, c_{0}$ related by $c_{n-1}=$ $c_{n}(2 n+d-2) /(2 n-1)$ such that

$$
c_{n}|x|^{2 n}=\sum_{i=1}^{N} a_{i}\left\langle x, u_{i}\right\rangle^{2 n}
$$

for $n=0, \ldots, k$. Note that $c_{k} \neq 0$ (because $2 k=\operatorname{deg} G$ ) and thus $c_{0} \neq 0$. By (7), this means $a_{1}+\cdots+a_{N} \neq 0$.

Put $x=u$ in (7) and integrate over $S_{d-1}$. After dividing by $\omega_{d-1}$ and using (3) with $x=u_{i}$, the result is

$$
c_{n}=\sum_{i=1}^{N} a_{i} \mu_{n}\left|u_{i}\right|^{2 n}=\left(a_{1}+\cdots+a_{N}\right) \mu_{n}
$$

for $n \leqslant k$. Using (3) and (7) with $x$ replaced by a unit vector $e$, (8) becomes

$$
\sum_{i=1}^{N} a_{i}\left\langle e, u_{i}\right\rangle^{2 n}=\left(a_{1}+\cdots+a_{n}\right) \int_{S_{d-1}}\langle e, u\rangle^{2 n} d \omega(u) .
$$

Taking $w_{i}=a_{i} /\left(a_{1}+\cdots+a_{N}\right)$ we see that (2) holds for $p(x)=\langle e, x\rangle^{2 n}, n \leqslant k$. Since such functions span the space of even polynomials of degree $\leqslant 2 k$, it follows by linearity that (2) holds for all $p$ in that space. Thus the necessary constants $w_{i}$ exist.

The extent to which the solution $h_{i}=w_{i} h_{G}$ of (1) is unique is made precise by

THEOREM 2. If $h$ is an even polynomial and $G(x)=\sum_{i=1}^{N} b_{i} h\left(\left\langle x, u_{i}\right\rangle\right)$ is radial, then $\left(b_{1}+\cdots+b_{N}\right) h=h_{G}$ where $h_{G}$ is obtained from $G$ as in Theorem 1 . If in addition, $G$ is not identically 0 then $b=b_{1}+\cdots+b_{N}$ is not 0 and the weights defined by $w_{i}=b_{i} / b$ make (2) valid for all even polynomials $p$ of degree $\leqslant \operatorname{deg} G$.

Proof. Equating the terms in $G(x)=\sum_{i=1}^{N} b_{i} h\left(\left\langle x, u_{i}\right\rangle\right)$ that are homogeneous of degree $2 n$ gives

$$
g_{n}|x|^{2 n}=\sum_{i=1}^{N} b_{i} s_{n}\left\langle x, u_{i}\right\rangle^{2 n}
$$

where $g_{n}$ is the coefficient of $|x|^{2 n}$ in $G(x)$ and $s_{n}$ is the coefficient of $t^{2 n}$ in $h(t)$. Take $x=u$ in (10) and integrate over $S_{d-1}$. After dividing by $\omega_{d-1}$ and using (3) with $x=u_{i}$, the result is

$$
g_{n}=\sum_{i=1}^{N} b_{i} s_{n} \mu_{n}\left|u_{i}\right|^{2 n}=\left(b_{1}+\cdots+b_{N}\right) s_{n} \mu_{n}
$$

which proves that $b h=h_{G}$. If $G \neq 0$ then $h_{G} \neq 0$ so $b \neq 0$. Let $2 k=\operatorname{deg} G$. Taking $n=k$ in (10) gives (6) with $a_{i}=s_{k} b_{i}$. By the argument given for Theorem 1, the weights $w_{i}=a_{i} /\left(a_{1}+\cdots+a_{N}\right)$ make (2) valid. Since $b_{i} / b$ gives the same weights, the proof is complete.

We close this paper by considering the distributional variant of (1) and by indicating how Theorem 1 generalizes. First we recall some notation and definitions. 
If $\Omega$ is an open subset of $R^{d}, d \geqslant 1, \mathscr{D}(\Omega)$ denotes the space of $C^{\infty}$ functions with compact support in $\Omega$ with the usual topology and $\mathscr{D}^{\prime}(\Omega)$ denotes its dual, the space of distributions on $\Omega$. If $\phi$ is in $\mathscr{D}(\Omega)$ and $T \in \mathscr{D}^{\prime}(\Omega),\langle T, \phi\rangle_{\Omega}$ denotes $T$ evaluated at $\phi$. Set $I_{1}=\{t \in R:-1<t<1\}$ and, again assuming $d \geqslant 2$, set $\Omega_{1}=\left\{x \in R^{d}\right.$ : $|x|<1\}$.

If $\phi$ is in $\mathscr{D}\left(\Omega_{1}\right)$ and $h$ is in $\mathscr{D}^{\prime}\left(I_{1}\right)$ take $Q_{u} \phi(t)$ to be the result of integrating $\phi$ over the hyperplane $\langle x, u\rangle=t$, notice that $Q_{u} \phi(t)$ is in $\mathscr{D}\left(I_{1}\right)$ as a function of $t$, call this function $Q_{u} \phi$, and define the distribution $1 \otimes_{u} h$ in $\mathscr{D}^{\prime}\left(\Omega_{1}\right)$ by the formula

$$
\left\langle 1 \otimes_{u} h, \phi\right\rangle_{\Omega_{1}}=\left\langle h, Q_{u} \phi\right\rangle_{I_{1}} .
$$

Observe that if $h$ is locally integrable on $I_{1}$ then $1 \otimes_{u} h$ is locally integrable on $\Omega$ and $1 \otimes_{u} h(x)=h(\langle x, u\rangle)$. Thus the distributional variant of $(1)$ is

$$
G=\sum_{i=1}^{N} 1 \otimes_{u_{i}} h_{i}
$$

where $G$ is in $\mathscr{D}^{\prime}\left(\Omega_{1}\right)$ and $h_{i}$ is in $\mathscr{D}^{\prime}\left(I_{1}\right), i=1, \ldots, N$.

To rule out obviously redundant terms in (1) and (12) we may assume

$$
u_{i} \neq-u_{j} \quad \text { and } \quad u_{i} \neq u_{j} \text { for } i \neq j .
$$

THEOREM 3. Let $G$ be in $\mathscr{D}^{\prime}\left(\Omega_{1}\right)$ and let $h_{1}, \ldots, h_{N}$ be in $\mathscr{D}^{\prime}\left(I_{1}\right)$. If $G$ is radial and (12) holds, then $G$ must be a polynomial. If (13) also holds, then $h_{1}, \ldots, h_{N}$ must be polynomials.

Proof. Representing directional differentiation in the direction $v$ by the usual notation $D_{v}$, we see that $Q_{u} D_{v} \phi=\langle u, v\rangle D Q_{u} \phi$. Hence if $h$ is in $\mathscr{D}^{\prime}\left(I_{1}\right)$, then $D_{v}\left(1 \otimes_{u} h\right)=\langle u, v\rangle\left(1 \otimes_{u} D h\right)$.

Choosing unit vectors $v_{1}, \ldots, v_{N}$ so that $\left\langle v_{i}, u_{i}\right\rangle=0$ for $i=1, \ldots, N$ we see that $D_{v_{1}} \cdots D_{v_{N}} G=0$. By Theorem 2 of [4] $G$ must be a polynomial. Because of (13), we can arrange the choice of $v_{2}, \ldots, v_{N}$ so that $c_{1}=\prod_{i=2}^{N}\left\langle v_{i}, u_{1}\right\rangle \neq 0$. Applying $D_{v_{2}}, \ldots, D_{v_{N}}$ to both sides of (13) we have

$$
D_{v_{2}} \cdots D_{v_{N}} G=c_{1}\left(1 \otimes_{u_{1}} D^{N-1} h_{1}\right) .
$$

Since $G$ is a polynomial, so is $F=D_{v_{2}} \cdots D_{v_{n}} G$. By (14) we see that $D_{v} F=0$ for all $v$ perpendicular to $u_{1}$. Thus, for all $|x|<1, F(x)=f\left(\left\langle x, u_{1}\right\rangle\right)$ where $f(t)=F\left(t u_{1}\right)$. Using (14) it is easy to verify that $f=D^{N-1} h_{1}$. Hence $h_{1}$ is a polynomial. Since similar arguments apply to $h_{2}, \ldots, h_{N}$, the proof is complete.

Combining Theorems 1 and 3 gives the following

COROLlARY. If (2) holds for all even polynomials of degree $\leqslant \nu$, then every radial polynomial $G$ of degree $\leqslant \nu$ enjoys a representation of the form (1). Conversely, if (12) holds where $G$ is a radial distribution in $\mathscr{D}^{\prime}\left(\Omega_{1}\right)$ and $h_{1}, \ldots, h_{N}$ are in $\mathscr{D}^{\prime}\left(I_{1}\right)$, then $G$ is a polynomial and there are constants $w_{1}, \ldots, w_{N}$ which make (2) valid for all even polynomials of degree $\leqslant \operatorname{deg} G$. 


\section{REFERENCES}

1. A. Erdelyi (ed.), Tables of integral transforms, McGraw-Hill, New York, 1954.

2. F. A. Grünbaum, The limited angle reconstruction problem, Computed Tomography (L. A. Shepp, ed.), Proc. Sympos. Appl. Math., Vol. 27, Amer. Math. Soc., Providence, R.I., 1983, pp. 43-61.

3. B. F. Logan and L. A. Shepp, Optimal reconstruction of a function from its projections, Duke Math. J. 42 (1975), 645-659.

4. W. R. Madych and S. A. Nelson, Characterization of tomographic reconstructions which commute with rigid motions, J. Funct. Anal. 46 (1982), 258-263.

5. __ Polynomial based algorithms for computed tomography. II, SIAM J. Appl. Math. 44 (1984), 193-208.

6. __ Radial sums of ridge functions: a characterization, Math. Methods Appl. Sci. 7 (1985), 90-100.

7. A. D. McLaren, Optimal numerical integration on a sphere, Math. Comp. 17 (1963), 361-383.

8. K. T. Smith, Reconstruction formulas in computed tomography, Computed Tomography (L. A. Shepp, ed.), Proc. Sympos. Appl. Math., Vol. 27, Amer. Math. Soc., Providence, R.I., 1983, pp. 7-23.

9. L. A. Shepp and J. B. Kruskal, Computerized tomography: the new medical X-ray technology, Amer. Math. Monthly 85 (1978), 420-439.

10. A. H. Stroud, Approximate calculation of multiple integrals, Prentice-Hall, Englewood Cliffs, N.J., 1971.

Department of Mathematics, University of Connecticut, Storrs, Connecticut 06268

Department of Mathematics, Iowa State University, Ames, Iowa 50011 